\title{
IFN drives synapse loss via microglia
}

Microglia are critical mediators of central nervous system (CNS) disease manifestations in systemic lupus erythematosus (SLE), according to new findings published in Nature. "In the field of SLE research, the mechanisms of CNS disease in SLE have been unclear," explains corresponding author Michael Carroll. "We identify type I interferon (IFN) as a modulator of microglia function that can stimulate synapse loss and microglia engulfment of synaptic material."

"While work by Betty Diamond and others have shown that autoantibodies can contribute to CNS lupus, these instances do not fully account for the high prevalence and variety of neuropsychiatric symptoms in patients with SLE," states Allison Bialas, first author of the paper.
The investigators characterized the behaviour of lupus-prone mice, finding that the behavioural phenotypes indicative of CNS disease could be blocked with administration of a type I interferon receptor (IFNAR)blocking antibody.

The lupus-prone mice had no signs of inflammation or cellular infiltration of the brain, suggesting the involvement of CNS-resident cells rather than infiltrating immune cells in CNS manifestations. Furthermore, quantitative PCR analysis revealed increased expression of Ifna and the IFN-stimulated gene $M x 1$ in the spleen of these mice, whereas in the brain only $M x 1$ expression was increased, indicating upregulation of IFNAR signalling in the brain by systemic type I IFN.

The most prevalent $M x 1$-positive cell type was microglia (resident macrophages of the brain). A higher proportion of $M x 1$-positive microglia had a reactive phenotype than $M \times 1$ negative microglia and indeed lupusprone mice had increased numbers of reactive microglia compared with their wild-type littermates, which could be reduced with anti-IFNAR treatment. Using bone marrow chimeras to separate the effects of peripheral inflammation, Bialas et al. found that the increased number of reactive microglia was dependent on IFNAR, but not on IFNAR expression by peripheral immune cells, supporting a direct effect of IFNAR on microglia.

Synapse density in the frontal cortex was lower in lupus-prone mice relative to wild-type mice; this synapse loss was reduced with anti-IFNAR treatment. To investigate the mechanism, lupus-prone mice were crossed to mice expressing a GFP-fusion protein that localizes throughout neurons, enabling visualization of neuronal material uptake. Using this model, microglia of lupus-prone mice were demonstrated to internalize more neuronal material than those of wild-type controls, a finding also confirmed by transmission electron microscopy imaging. This engulfment correlated with $M x 1$ expression, and was decreased with anti-IFNAR treatment and stimulated with biotinylated IFN $\alpha$ or IFN $\beta$ treatment.

"Going forward, we are working to understand whether changes in neurons stimulate microglia to target synapses or whether microglia are truly initiating this process," says Carroll. "We are also investigating whether synapse loss in lupus-prone mice is complement-dependent or whether other phagocytic pathways have a role and, finally, how this mechanism might fit in with antibody-mediated damage to the CNS."

"This study offers new insights into neuropsychiatric lupus," states Betty Diamond, who was not involved in the study. "It will be important to pursue these observations to learn if IFN also has direct toxic effects on neurons in lupus models and whether IFN-blockade leads to a reversal, not just a prevention, of behavioural phenotypes".

Although phase III clinical trials of anti-IFNAR therapies are currently underway, Bialas points out that these trials do not include patients with CNS symptoms or indeed include such symptoms in their clinical end points. "We hope that our work can be useful in designing future trials focused on CNS lupus," Bialas concludes.

Jessica McHugh

ORIGINAL ARTICLE Bialas, A.et al. Microgliadependent synapse loss in type I interferonmediated lupus. Nature http://dx.doi.org/10.1038/ nature22821 (2017) 